Published in final edited form as:

Neuropharmacology. 2015 January ; 88: 1. doi:10.1016/j.neuropharm.2014.10.001.

\title{
GABAergic Signaling in Health and Disease
}

Chris J. McBain ${ }^{*}, 1$, Josef Kittler ${ }^{1}$, Bernhard Luscher ${ }^{1}$, Istvan Mody ${ }^{1}$, and Beverley A. Orser ${ }^{1}$ Porter Neuroscience Research Center, Eunice Kennedy Shriver National Institute of Child Health and Human Development, Building 35, Room 3C-903, 35 Convent Drive, MSC 3715, Bethesda, MD, 20892-3715, United States

This Special Issue of Neuropharmacology on the topic of GABAergic Signaling in Health and Disease was organized to accompany the highly successful 24th Neuropharmacology, Elsevier Conference that took place in Arlington Virginia, USA on the 13-14 November, 2014. As part of this conference all speakers and attendees were invited to submit original research articles or reviews pertinent to the topic covered in the Conference for consideration in this Special Issue of Neuropharmacology. As you will see from the content inside, the response was tremendous, with a highly diverse range of topics covered. This snapshot of research interests illustrates both recent advances and the current state of this rich field of the Neurosciences.

The last decade has seen an explosion of research targeted towards the physiological and pathophysiological roles played by local circuit inhibitory interneurons, the neurotransmitter GABA and its ionotropic and metabotropic receptors. Recent advances in novel molecular, genetic and optogenetic approaches have propelled this research field rapidly forward in ways that it would have been hard to imagine only a few years ago. The conference on GABAergic Signaling in Health and Disease bore witness to the high quality of research currently being performed and the meeting's content is mirrored in articles presented in this Special Issue. In only a few years the topic of "GABAergic Signaling" has broken free from its original confines of pharmacology and now comfortably extends to also cover diverse topics such as the structure, function and assembly of different subtypes of $\mathrm{GABA}_{\mathrm{A}}$ and $\mathrm{GABA}_{\mathrm{B}}$ receptors, how GABA transporters shape transmitter availability and network excitability, the roles of GABA receptors in neuronal development and plasticity and the dependence of oscillatory and circuit phenomena on particular inhibitory interneurons and differential GABA receptor expression; all of these topics are covered by articles in this Special issue.

Recent research highlights have revolutionized our thinking about the role of GABA and GABAergic systems. At the cellular level, the functional importance of $\mathrm{GABA}_{\mathrm{A}}$ receptors that reside outside synapses has been revealed. These "extrasynaptic" receptors differ from both pre- and postsynaptic $\mathrm{GABA}_{\mathrm{A}}$ receptors as they are activated in a paracrine manner by low ambient levels of GABA. At the network level, GABA is no longer viewed simply as an inhibitory neurotransmitter that dampens neuronal excitability. Rather, the synchronization

"Corresponding author: mcbainc@mail.nih.gov (C.J. McBain).

${ }^{1}$ Organizers of the 24 th Neuropharmacology Conference on GABAergic Signaling in Health and Disease. 
of network activity is finely tuned by a diverse array of GABAergic interneurons, each with distinct intrinsic and firing properties that uniquely influence neural networks. Particularly promising is extension of this field into many divergent neurodegenerative, neuropsychiatric and pathophysiological conditions. Once considered only within the confines of the epilepsies, GABA receptors and inhibitory interneurons are now recognized to play key roles in virtually all central nervous system disorders. Further, different subtypes of GABA receptors with distinct physiological and pharmacological properties are being pursued as promising targets for drug development. These translational discoveries are reaping the reward of decades of research into interneurons and GABA receptors laid down by many excellent research labs. Finally, $\mathrm{GABA}_{\mathrm{A}}$ receptors are widely expressed outside the central nervous system in the lung, liver, pancreas and other organ systems. The physiological and pathophysiological properties and the in vivo roles of these receptors are only beginning to be understood.

We hope that in reading this Special Issue that you will agree that it is an exciting time for all aspects of GABAergic Signaling within the central nervous system and we expect that the next decade will reveal many further secrets regarding this diverse and exciting field. 